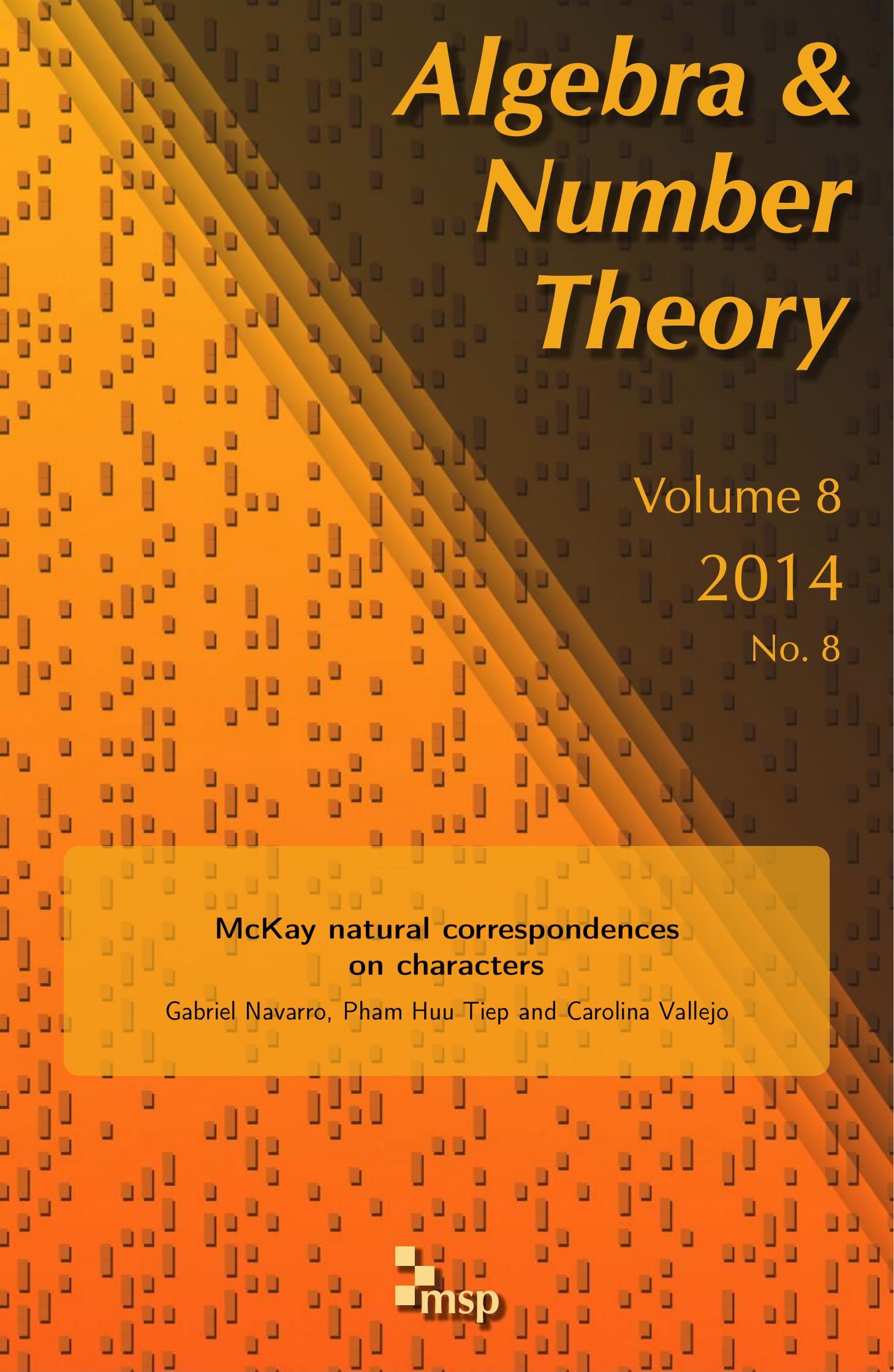




\title{
McKay natural correspondences on characters
}

\author{
Gabriel Navarro, Pham Huu Tiep and Carolina Vallejo
}

Let $G$ be a finite group, let $p$ be an odd prime, and let $P \in \operatorname{Syl}_{p}(G)$. If $N_{G}(P)=P C_{G}(P)$, then there is a canonical correspondence between the irreducible complex characters of $G$ of degree not divisible by $p$ belonging to the principal block of $G$ and the linear characters of $P$. As a consequence, we give a characterization of finite groups that possess a self-normalizing Sylow $p$-subgroup or a $p$-decomposable Sylow normalizer.

\section{Introduction}

The McKay conjecture, one of the main problems in the representation theory of finite groups, asserts that if $G$ is a finite group and $P$ is a Sylow $p$-subgroup of $G$, then $\left|\operatorname{Irr}_{p^{\prime}}(G)\right|=\left|\operatorname{Irr}_{p^{\prime}}\left(N_{G}(P)\right)\right|$, where $\operatorname{Irr}_{p^{\prime}}(G)$ is the set of the irreducible complex characters of $G$ that have degree not divisible by $p$. It is well known that, in general, no choice-free correspondence can exist between $\operatorname{Irr}_{p^{\prime}}(G)$ and $\operatorname{Irr}_{p^{\prime}}\left(N_{G}(P)\right)$. (On the other hand, the existence of certain type of bijections between these two sets is the idea on which a possible solution of the McKay conjecture is nowadays based [Isaacs et al. 2007].)

A key case to consider and understand in the McKay conjecture is when $P$ is self-normalizing or, even, when $N_{G}(P)=P C_{G}(P)$. It is not often that a natural correspondence of characters is found.

Theorem A. Let $G$ be a finite group, let $p$ be odd, and let $P \in \operatorname{Syl}_{p}(G)$. Suppose that $N_{G}(P)=P C_{G}(P)$. If $\chi \in \operatorname{Irr}_{p^{\prime}}(G)$ lies in the principal block, then

$$
\chi_{N_{G}(P)}=\chi^{*}+\Delta,
$$

where $\chi^{*} \in \operatorname{Irr}\left(N_{G}(P)\right)$ is linear in the principal block and $\Delta$ is either zero or a character whose irreducible constituents all have degree divisible by p. Furthermore,

The research of Navarro and Vallejo is partially supported by the Spanish Ministerio de Educación y Ciencia proyecto MTM2013-40464-P. Tiep gratefully acknowledges the support of the NSF (grants DMS-0901241 and DMS-1201374) and the Simons Foundation Fellowship 305247.

MSC2010: primary 20C15; secondary 20C20.

Keywords: McKay conjecture, self-normalizing Sylow subgroup, $p$-decomposable Sylow normalizer. 
the map $\chi \mapsto \chi^{*}$ is a bijection

$$
\operatorname{Irr}_{p^{\prime}}\left(B_{0}(G)\right) \rightarrow \operatorname{Irr}_{p^{\prime}}\left(B_{0}\left(N_{G}(P)\right)\right),
$$

where $\operatorname{Irr}_{p^{\prime}}\left(B_{0}(G)\right)$ is the set of irreducible characters in the principal block of $G$ of degree not divisible by $p$.

For $p=2$, Theorem A is unfortunately false, as shown, for instance, by $\mathrm{S}_{5}$. (To prove the McKay conjecture for $p=2$ for groups with a self-normalizing Sylow $p$-subgroup is still a challenge.) For $p$ odd, Theorem $\mathrm{A}$ is also not true for $p$-blocks of maximal defect, as shown by the following example: $G=\mathrm{SL}_{2}(27) \cdot C_{3}$ has a rational, faithful, irreducible character $\chi$ of degree 26 that belongs to the unique nonprincipal 3-block of maximal defect of $G$, and $\chi_{N_{G}(P)}$ contains two linear characters as irreducible constituents.

Theorem A yields the following immediate consequence.

Corollary B. Let $G$ be a finite group, let $p$ be odd, and let $P \in \operatorname{Syl}_{p}(G)$. Suppose that $N_{G}(P)=P$. Then there is a natural bijection $\chi \mapsto \chi^{*}$ between $\operatorname{Irr}_{p^{\prime}}(G)$ and the linear characters of $P$. In fact, if $\chi \in \operatorname{Irr}_{p^{\prime}}(G)$ and $\lambda \in \operatorname{Irr}(P)$ is linear, then $\chi$ and $\lambda$ correspond under the bijection if and only if

$$
\chi_{P}=\lambda+\Delta,
$$

where $\Delta$ is either zero or a character whose irreducible constituents all have degree divisible by $p$. This happens if and only if

$$
\lambda^{G}=\chi+\Xi,
$$

where $\Xi$ is either zero or a character whose irreducible constituents all have degree divisible by $p$.

Corollary B was proved in [Navarro 2003] for $p$-solvable groups (although a different proof was later given in [Isaacs and Navarro 2008]).

We now mention several applications. A not very well-known consequence of the Galois version of the McKay conjecture [Navarro 2004] states that whenever $G$ is a finite group and $p$ is an odd prime, then $N_{G}(P)=P C_{G}(P)$ if and only if the principal character $1_{G}$ is the unique $p$-rational $p^{\prime}$-degree character in the principal block of $G$. If $N_{G}(P)=P C_{G}(P)$, it follows by Theorem A that the fields of values of the $p^{\prime}$-degree nontrivial irreducible characters in the principal block are cyclotomic fields $\mathbb{Q}_{p^{a}}$ for $a>0$, which implies one half of the statement above. (The other half will be treated separately in another paper.)

A consequence of Theorem A and Corollary B is the following (perhaps surprising) characterization of finite groups that possess a self-normalizing Sylow $p$-subgroup or a $p$-decomposable Sylow normalizer (i.e., $N_{G}(P)=P C_{G}(P)$ ), for a given odd prime $p$. 
Corollary C. Let $G$ be a finite group, let $p$ be odd, and let $P \in \operatorname{Syl}_{p}(G)$.

(a) $N_{G}(P)=P$ if and only if

$$
\left(1_{P}\right)^{G}=1_{G}+\Xi
$$

where $\Xi$ is either zero or a character whose irreducible constituents all have degree divisible by $p$.

(b) $N_{G}(P)=P C_{G}(P)$ if and only if $1_{G}$ is the only irreducible constituent of $\left(1_{P C_{G}(P)}\right)^{G}$ that has $p^{\prime}$-degree and belongs to the principal p-block of $G$.

It is remarkable that Corollary $\mathrm{C}(\mathrm{a})$ gives the exact opposite of a recent result by G. Malle and Navarro [2012]: a finite group $G$ has a normal Sylow $p$-subgroup if and only if all irreducible constituents of $\left(1_{P}\right)^{G}$ have degree not divisible by $p$.

Corollary $\mathrm{C}$ is false for $p=2$, as shown again by $G=\mathrm{S}_{5}$ : in this case $\left(1_{P}\right)^{G}$ contains the trivial character of $G$ and an irreducible character of degree 5 .

Now, we come back to Theorem A and natural correspondences. Although it is entirely possible that, under the hypotheses of Theorem A, a natural correspondence exists between all the characters in $\operatorname{Irr}_{p^{\prime}}(G)$ and $\operatorname{Irr}_{p^{\prime}}\left(N_{G}(P)\right.$ ) (not only the characters in the principal blocks), we have not been able to find it, except for $p$-solvable groups. In this case, our correspondence in Theorem $\mathrm{D}$ below extends the Glauberman correspondence (and the correspondence in Theorem A).

Theorem D. Let $G$ be a finite p-solvable group, and let $P \in \operatorname{Syl}_{p}(G)$. Suppose that $\boldsymbol{N}_{G}(P)=P \boldsymbol{C}_{G}(P)$, and let $N=\boldsymbol{O}_{p^{\prime}}(G)$. Let $\operatorname{Irr}_{P}(N)$ be the set of $P$-invariant characters $\theta \in \operatorname{Irr}(N)$. Then, for every $\theta \in \operatorname{Irr}_{P}(N)$ and linear $\lambda \in \operatorname{Irr}\left(P / P^{\prime}\right)$, there is a canonically defined character

$$
\lambda \star \theta \in \operatorname{Irr}_{p^{\prime}}(G) .
$$

Furthermore, the map

$$
\operatorname{Irr}\left(P / P^{\prime}\right) \times \operatorname{Irr}_{P}(N) \rightarrow \operatorname{Irr}_{p^{\prime}}(G)
$$

given by $(\lambda, \theta) \mapsto \lambda \star \theta$ is a bijection. As a consequence, $N_{G}(P)=P \times C_{N}(P)$, and if $\theta^{*} \in \operatorname{Irr}\left(\boldsymbol{C}_{N}(P)\right)$ is the Glauberman correspondent of $\theta \in \operatorname{Irr}_{P}(N)$, then the map

$$
\lambda \times \theta^{*} \mapsto \lambda \star \theta
$$

is a natural bijection $\operatorname{Irr}_{p^{\prime}}\left(N_{G}(P)\right) \rightarrow \operatorname{Irr}_{p^{\prime}}(G)$. Also, if $\theta=1_{N}$ and $\lambda \in \operatorname{Irr}\left(P / P^{\prime}\right)$, then $\lambda \times \theta^{*}$ is the unique linear constituent of $(\lambda \star \theta)_{N_{G}(P)}$.

Theorem D suggests studying the blocks $B$ of finite groups with defect group $D$ satisfying $N_{G}\left(D, b_{D}\right)=D C_{G}(D)$, where $\left(D, b_{D}\right)$ is a root of $B$. However, we will leave this for another place. 


\section{An extension theorem}

We begin with a well-known lemma. If $N \triangleleft G$ and $\theta \in \operatorname{Irr}(N)$, then $I_{G}(\theta)$ denotes the stabilizer of $\theta$ in $G$. In general, we follow the notation of [Isaacs 2006] for characters. If $G$ is a finite group, $\operatorname{Irr}_{p^{\prime}}(G)$ denotes the set of the irreducible complex characters of $G$ whose degree $\chi(1)$ is not divisible by the prime $p$.

Lemma 2.1. Let $G$ be a finite group, let $p$ be a prime, let $P \in \operatorname{Syl}_{p}(G)$, and let $\chi \in \operatorname{Irr}_{p^{\prime}}(G)$. Assume that $L \triangleleft G$. Then $\chi_{L}$ has a P-invariant irreducible constituent $\theta$, and all such constituents are $N_{G}(P)$-conjugate. In particular, if $N_{G / L}(P L / L)=P L / L$, then $\theta$ is unique.

Proof. Let $\eta \in \operatorname{Irr}(L)$ be any irreducible constituent, and let $T$ be the inertia subgroup of $\eta$ in $G$. By the Clifford correspondence, $|G: T|$ is not divisible by $p$, and therefore $P^{g^{-1}} \leq T$ for some $g \in G$, and thus $P$ fixes $\eta^{g}=\theta$. If $P$ fixes $\theta^{x}$, then $P^{x^{-1}}$ and $P$ are Sylow $p$-subgroups of $I=I_{G}(\theta)$, and $P^{y}=P^{x^{-1}}$ for some $y \in I$. Hence $y x \in N_{G}(P)$ and $\theta^{x}=\theta^{y x}$. The second part easily follows.

Lemma 2.2. Let $G$ be a finite group, let $p$ be prime, and let $P \in \operatorname{Syl}_{p}(G)$. Let $L \triangleleft G$, and assume that $N_{G / L}(P L / L)=P L / L$. Let $\theta \in \operatorname{Irr}(L)$ be $P$-invariant, let $T=$ $I_{G}(\theta)$ be the stabilizer of $\theta$ in $G$ and assume that $\psi \in \operatorname{Irr}(T \mid \theta)$ has $p^{\prime}$-degree. Then

$$
\left(\psi^{G}\right)_{L P}=\psi_{L P}+\Delta,
$$

where either $\Delta=0$ or every irreducible constituent of $\Delta$ has degree divisible by $p$. Proof. Let

$$
G=\bigcup_{x \in D} T x P
$$

be a disjoint union of double cosets with $1 \in D$. Then, by Mackey's formula, we have that

$$
\left(\psi^{G}\right)_{L P}=\psi_{L P}+\sum_{1 \neq x \in D}\left(\left(\psi^{x}\right)_{T^{x} \cap L P}\right)^{L P}
$$

Suppose that some irreducible constituent $\alpha$ of $\left(\left(\psi^{x}\right)_{T^{x} \cap L P}\right)^{L P}$ has degree not divisible by $p$ for $1 \neq x \in D$. Hence $\alpha_{L} \in \operatorname{Irr}(L)$ by Corollary (11.29) of [Isaacs 2006]. Thus the irreducible character $\alpha_{T^{x} \cap L P}$ lies under $\psi^{x}$. However $\left(\psi^{x}\right)_{L}=d \theta^{x}$, so we conclude that $\theta^{x}=\alpha_{L}$ is $P$-invariant. Then by Lemma 2.1, we have that $\theta^{x}=\theta$ and therefore $x \in T$. But this is impossible since $1 \neq x \in D$ is a representative of the double cosets of $T$ and $P$ in $G$.

The following theorem is key in this paper, and follows from deep results in [Navarro and Späth 2014] and [Späth 2013] on the McKay conjecture. Despite many efforts, we have been unable to find an elementary proof of it. Recall that a finite simple group $X$ is involved in a finite group $G$ if there exist $K \triangleleft H \leq G$ such 
that $X \cong H / K$. The so-called inductive Alperin-McKay condition is defined in Definition 7.2 of [Späth 2013]. For character triples, see Chapter 11 of [Isaacs 2006].

Theorem 2.3. Let $G$ be a finite group, and let $p$ be a prime. Let $P \in \operatorname{Syl}_{p}(G)$ and assume that $P=N_{G}(P)$. Let $L \triangleleft G$ and let $\theta \in \operatorname{Irr}(L)$ be $P$-invariant of $p^{\prime}$-degree. Suppose that $L \triangleleft H$ with $H / L$ a $p^{\prime}$-group. Assume that all nonabelian simple groups of order divisible by $p$ involved in $L$ satisfy the inductive Alperin-McKay condition for $p$. If $\theta$ is $H$-invariant, then $\theta$ extends to $H$. In particular, this holds if $p$ is odd.

Proof. We argue by induction on $|G|$. Let $Q=P \cap L$. We are going to use Theorem 7.1 of [Navarro and Späth 2014]. The notation $\operatorname{Irr}_{0}(L \mid Q)$ in that theorem is defined in Notation 2.1 of the same article, and since $Q \in \operatorname{Syl}_{p}(L)$, we have that $\operatorname{Irr}_{0}(L \mid Q)=\operatorname{Irr}_{p^{\prime}}(L)$ in this case. Theorem 7.1 of [loc. cit.] implies now that there is a $N_{G}(Q)$-equivariant bijection

$$
\Pi_{Q}: \operatorname{Irr}_{p^{\prime}}(L) \rightarrow \operatorname{Irr}_{p^{\prime}}\left(N_{L}(Q)\right)
$$

such that the character triples $(T, L, \theta)$ and $\left(N_{T}(Q), N_{L}(Q), \theta^{\prime}\right)$ are isomorphic, where $\theta^{\prime}=\Pi_{Q}(\theta)$ and $T=I_{G}(\theta)$. (In Section 3 of [loc. cit.] the reader will find the appropriate definitions involved in Theorem 7.1 there.) Since $\Pi_{Q}$ is $N_{G}(Q)$ equivariant, we have that $N_{T}(Q)=I_{N_{G}(Q)}\left(\theta^{\prime}\right)$. Since $P \leq N_{G}(Q)$, we have that $\theta^{\prime}$ is $P$-invariant. By character triple isomorphisms, we have that $\theta$ extends to $H$ if and only if $\theta^{\prime}$ extends to $N_{H}(Q)$. Also $N_{H}(Q) / N_{L}(Q)$ is a $p^{\prime}$-group, so, arguing by induction, it is no loss to assume that $Q \triangleleft G$. Since $N_{G}(P)=P$, it follows that $C_{L / Q}(P)=1$. Let $\eta \in \operatorname{Irr}(Q)$ be $P$-invariant under $\theta$, by Theorem (13.27) of [Isaacs 2006]. Let $I=I_{G}(\eta)$. Since $\theta$ is $H$-invariant, we have that $H=L(I \cap H)$ by using Clifford's theorem. Let $\tau \in \operatorname{Irr}(I \cap L \mid \eta)$ be the Clifford correspondent of $\theta$ over $\eta$. By the uniqueness in the Clifford correspondence, we have that $\tau$ is $I \cap H$-invariant. If $I<G$, then by induction we have that $\tau$ has an extension $\rho \in \operatorname{Irr}(I \cap H)$. Now,

$$
\left(\rho^{H}\right)_{L}=\left(\rho_{I \cap L}\right)^{L}=\epsilon^{L}=\theta,
$$

and we are also done in this case. So we may assume that $\eta$ is $G$-invariant. Since $C_{L / Q}(P)=1$, by Problem (13.10) of [loc. cit.] $\theta$ is the unique $P$-invariant constituent of $\eta^{L}$. Now, we have that $\eta$ has an extension $\hat{\eta} \in \operatorname{Irr}(H)$ by Corollary (8.16) of [loc. cit.]. Since $(\hat{\eta})_{L}$ is $P$-invariant and lies over $\eta$, it coincides with $\theta$ by uniqueness. Hence $\theta$ extends to $H$, as required.

If $p$ is odd, then by Theorem A of [Guralnick et al. 2004], we have that all nonabelian composition factors of $G$ of order divisible by $p$ are $\operatorname{PSL}_{2}\left(3^{3^{a}}\right)$ with $a \geq 1$ and that $p=3$. By elementary general group theory, if $X$ is a simple group involved in $G$, then $X$ is involved in a composition factor of $G$. By using the classification of the subgroups of $\mathrm{PSL}_{2}\left(p^{f}\right)$ in Satz II.8.27 of [Huppert 1967], we have that the only simple groups involved in $G$ of order divisible by $p$ are $\operatorname{PSL}_{2}\left(3^{3^{b}}\right.$ ) (with $p=3$ 
and $b \geq 1$ ). Now, the proof of Theorem 8.4 of [Späth 2013] shows that the simple groups $\mathrm{PSL}_{2}\left(3^{3^{b}}\right)$ with $b \geq 1$ satisfy the inductive Alperin-McKay condition.

Corollary 2.4. Let $G$ be a finite group, $p$ any prime, $P \in \operatorname{Syl}_{p}(G)$, and assume that $P=N_{G}(P)$. Let $L \triangleleft G$. Let $\chi \in \operatorname{Irr}_{p^{\prime}}(G)$, and let $\theta \in \operatorname{Irr}(L)$ be P-invariant under $\chi$. Assume that all nonabelian simple groups of order divisible by $p$ involved in L satisfy the inductive Alperin-McKay condition for $p$. Then $\theta$ extends to $I_{G}(\theta)$. In particular, this holds if $p$ is odd.

Proof. We may assume that $\theta$ is $G$-invariant. Now, $\chi_{P L}$ has some irreducible constituent $\xi \in \operatorname{Irr}(P L)$ such that $p$ does not divide $\xi(1)$. Then $\xi_{L}=\theta$, by Corollary (11.29) of [Isaacs 2006]. Suppose now that $q \neq p$ is another prime, and let $Q / L$ be a Sylow $q$-subgroup of $G / L$. Then $\theta$ extends to $Q$ by Theorem 2.3. Hence, we have that $\theta$ extends to $G$ by Corollary (11.30) of [loc. cit.].

\section{A group theoretical result}

Our aim in this Section is to prove Theorem 3.2 below. We start with the following lemma, whose parts (ii) and (iii) will be used in the proof of Theorem A.

Lemma 3.1. Let $S:=\operatorname{PSL}_{2}(q)$ with $q=3^{3^{a}}$ for some $a \geq 1, P \in \operatorname{Syl}_{3}(\operatorname{Aut}(S))$, and $Q:=P \cap S \in \operatorname{Syl}_{3}(S)$.

(i) Assume that $Y$ is a $3^{\prime}$-subgroup of $\operatorname{Aut}(S)$ that centralizes $Q$. Then $Y=1$.

(ii) Assume that $Q \leq R \leq P$ and $C_{N_{S}(Q) / Q}(R)=1$. Then $R=P$.

(iii) $\operatorname{Irr}(S)$ contains exactly four $P$-invariant characters: the principal character $1_{S}$, two irreducible Weil characters $\eta^{ \pm}$of degree $(q-1) / 2$, and the Steinberg character of degree $q$. If $\alpha \in\left\{1_{S}, \eta^{+}, \eta^{-}\right\}$, then $\alpha_{Q}$ contains a unique P-invariant irreducible constituent $\alpha^{*}$, which occurs with multiplicity one. Finally, the map $\alpha \mapsto \alpha^{*}$ is a bijection between $\left\{1_{S}, \eta^{+}, \eta^{-}\right\}$and the set of $P$-invariant irreducible characters of $Q$.

Proof. (i) Recall that $\operatorname{Aut}(S) \cong \operatorname{PGL}_{2}(q) \cdot C_{3^{a}}$. Since $Y$ is a $3^{\prime}$-group, it embeds in $C_{H}(Q)$ for $H:=\operatorname{PGL}_{2}(q)$. But $\boldsymbol{C}_{H}(Q)=Q$, hence the claim follows.

(ii) Without loss we may assume that $Q$ is the image of the subgroup

$$
\left\{[x]:=\left(\begin{array}{ll}
1 & x \\
0 & 1
\end{array}\right) \mid x \in \mathbb{F}_{q}\right\}
$$

in $\operatorname{PSL}_{2}(q)$ and $P=\langle Q, \sigma\rangle$, where $\sigma$ acts on $S$ as the field automorphism raising every entry $y$ of any matrix in $\mathrm{SL}_{2}(q)$ to $y^{3}$. Then the maximal subgroup $\left\langle Q, \sigma^{3}\right\rangle$ of $P$ centralizes a subgroup of order 13 of $N_{S}(Q) / Q$, namely, the one induced by $\left\{\operatorname{diag}\left(z, z^{-1}\right) \mid z \in \mathbb{F}_{27}^{\times}\right\}$. Hence the claim follows. 
(iii) We keep the notation of (ii). The character table of $S$ is given, for instance, in [Digne and Michel 1991, Table 2]. Now, it is straightforward to check that $1_{S}, \eta^{ \pm}$, and the Steinberg character (of degree $q$ ) are the only $P$-invariant irreducible characters of $S$. Next,

$$
\operatorname{Irr}(Q)=\left\{\lambda_{a}:[x] \rightarrow \omega^{\operatorname{tr}_{\mathbb{F}_{q} / \mathbb{F}_{3}}(a x)} \mid a \in \mathbb{F}_{q}\right\},
$$

where $\omega \in \mathbb{C}$ is a fixed primitive cubic root of unity. Since $\sigma$ acts on $\operatorname{Irr}(Q)$ via $\lambda_{a} \mapsto \lambda_{a^{3}}$, the only $P$-invariant irreducible characters of $Q$ are $1_{Q}=\lambda_{0}, \lambda_{1}$, and $\lambda_{-1}$. Relabeling $\eta^{+}$and $\eta^{-}$if necessary, we have that

$$
\left(\eta^{+}\right)_{Q}=\sum_{a \in \mathbb{F}_{q}^{\times 2}} \lambda_{a}, \quad\left(\eta^{-}\right)_{Q}=\sum_{a \in \mathbb{F}_{q}^{\times} \backslash \mathbb{F}_{q}^{\times 2}} \lambda_{a} .
$$

Hence $\lambda_{1}$ and $\lambda_{-1}$ are the only $P$-invariant irreducible constituents of $\left(\eta^{+}\right)_{Q}$ and $\left(\eta^{-}\right)_{Q}$, respectively, each occurring with multiplicity one.

Theorem 3.2. Let $G$ be a finite group, let $p$ be a prime, and let $P \in \operatorname{Syl}_{p}(G)$. Suppose that $\boldsymbol{N}_{G}(P)=P \times X$. If $p$ is odd or $G$ is p-solvable, then $X \leq \boldsymbol{O}_{p^{\prime}}(G)$. In particular, if $\boldsymbol{N}_{G}(P)=P \boldsymbol{C}_{G}(P)$, then $\boldsymbol{O}_{p^{\prime}}\left(\boldsymbol{N}_{G}(P)\right) \leq \boldsymbol{O}_{p^{\prime}}(G)$.

Proof. We argue by induction on $|G|$. If $N \triangleleft G$, then

$$
N_{G / N}(P N / N)=N_{G}(P) N / N=P N / N \times X N / N .
$$

Hence, if $N>1$, then we have that $X N / N \leq \boldsymbol{O}_{p^{\prime}}(G / N)$. In particular, we may assume that $\boldsymbol{O}_{p^{\prime}}(G)=1$. Now, suppose that $N=\boldsymbol{O}_{p}(G)>1$. Then we conclude that $X \leq \boldsymbol{O}_{p p^{\prime}}(G)=M$. Since $[X, P]=1$, then $[X, N]=1$. However, using that $\boldsymbol{O}_{p^{\prime}}(G)=1$, we have that $\boldsymbol{C}_{M}(N)=\boldsymbol{Z}(N) \times \boldsymbol{O}_{p^{\prime}}(M)=\boldsymbol{Z}(N)$, and we conclude that $X=1$, in this case. Hence, we may assume that $G$ is not $p$-solvable, and that $p$ is odd.

Now, let $N$ be a minimal normal subgroup of $G$. By [Guralnick et al. 2004], we have that $N=S_{1} \times \cdots \times S_{k}$, where $\left\{S_{1}, \ldots, S_{k}\right\}$ are transitively permuted by $G$ and $S_{1}=S=\operatorname{PSL}_{2}\left(3^{3^{a}}\right)$. Now $P \cap N=\left(P \cap S_{1}\right) \times \cdots \times\left(P \cap S_{k}\right)$. Fix some index $i$. Since $[P, X]=1$, we have $\left[Q_{i}, X\right]=1$, where $1<Q_{i}=P \cap S_{i} \in \operatorname{Syl}_{3}\left(S_{i}\right)$. Now, if $x \in X$, then we have that $\left(S_{i}\right)^{x}=S_{j}$ for some $j$. However $Q_{i}^{x} \leq S_{i}^{x} \cap S_{i}=S_{j} \cap S_{i}$, so we conclude that $X \leq N_{G}\left(S_{i}\right)$ for all $i$ with $\left[X, Q_{i}\right]=1$. Let $Y_{i}=X \boldsymbol{C}_{G}\left(S_{i}\right) / \boldsymbol{C}_{G}\left(S_{i}\right)$, which is a $3^{\prime}$-subgroup of $\operatorname{Aut}\left(S_{i}\right)$ centralizing the Sylow 3-subgroup $Q_{i}$ of $S_{i}$. By Lemma 3.1(i), $Y_{i}=1$, whence $X \leq \boldsymbol{C}_{G}\left(S_{i}\right)$ for all $i$. Thus $X \leq \boldsymbol{C}_{G}(N)$ for every minimal normal subgroup. Since $\boldsymbol{F}(G)=1$, we have $\boldsymbol{F}^{*}(G)=\boldsymbol{E}(G)=E$. Since $\boldsymbol{Z}(E)=1$, we have that $E$ is semisimple and $\boldsymbol{C}_{G}(E)=1$ by Theorems 9.7 and 9.8 of [Isaacs 2008]. Now, $E$ is a direct product of nonabelian simple groups $K_{i}$ and the normal closure of $K_{i}$ is a minimal normal subgroup of $G$ (by Lemma 9.17 of [Isaacs 2008], for instance), and we conclude that $X \leq \boldsymbol{C}_{G}(E)=1$, as desired. 
Finally, since $\boldsymbol{C}_{G}(P)=\boldsymbol{Z}(P) \times \boldsymbol{O}_{p^{\prime}}\left(\boldsymbol{N}_{G}(P)\right)$ (by the Schur-Zassenhaus theorem), it follows that if $N_{G}(P)=P C_{G}(P)$, then $N_{G}(P)=P \times \boldsymbol{O}_{p^{\prime}}\left(N_{G}(P)\right)$, and we apply the first part of the theorem.

Note that Theorem 3.2 is not true for $p=2$ : If $G=E_{6}(11)$ and $P \in \operatorname{Syl}_{2}(G)$, then $N_{G}(P)=P \times C_{5}$; see [Kondratiev and Mazurov 2003, Theorem 6(c)].

\section{Proof of main theorem}

We will also need the following result.

Lemma 4.1 [Navarro et al. 2007]. Suppose that a finite p-group $P$ acts on a finite group $G$, stabilizing $N \triangleleft G$. Suppose that $Q / N \in \operatorname{Syl}_{p}(G / N)$ is $P$-invariant, and assume that

$$
G / N=T_{1} / N \times \cdots \times T_{a} / N,
$$

where the subgroups $T_{1}, \ldots, T_{a}$ are permuted by $P$. Let $Q_{1}=Q \cap T_{1}$, and let $P_{1}$ be the stabilizer of $T_{1}$ in $P$. If $\boldsymbol{C}_{\boldsymbol{N}_{G}(Q) / Q}(P)=1$, then $\boldsymbol{C}_{\boldsymbol{N}_{T_{1}}\left(Q_{1}\right) / Q_{1}}\left(P_{1}\right)=1$.

Proof. This follows by applying Lemma 4.1 of [Navarro et al. 2007] to each of the $P$-orbits on $\left\{T_{1}, \ldots, T_{a}\right\}$.

The proof of the following lemma is a trivial consequence of the fact that $\boldsymbol{O}_{p^{\prime}}(G)$ is contained in the kernel of all the irreducible characters in the principal block of $G$. (See, for instance, Theorem (6.10) of [Navarro 1998].)

Lemma 4.2. Suppose that $N$ is a normal subgroup of $H$, with $N \leq \boldsymbol{O}_{p^{\prime}}(H)$. Suppose that $H=N U$ for some $U \leq H$. Then restriction defines a bijection between the characters of the principal block of $H$ and of the principal block of $U \cap N$.

We are finally ready to prove the main result of this paper. The only way we have found to prove it is to use a strong induction using normal subgroups. Theorem A of the introduction will be recovered by letting $L=1$ in the next result.

Theorem 4.3. Let $G$ be a finite group, and let $p$ be an odd prime. Let $P \in \operatorname{Syl}_{p}(G)$, and suppose that $N_{G}(P)=P C_{G}(P)$. Let $L \triangleleft G$. Let $\chi \in \operatorname{Irr}_{p^{\prime}}(G)$ lie in the principal block of $G$. Then $\chi_{L N_{G}(P)}=\chi^{*}+\Delta$, where $\chi^{*} \in \operatorname{Irr}_{p^{\prime}}\left(L N_{G}(P)\right)$ lies in the principal block of $L N_{G}(P)$, and $\Delta$ is either zero or a character of $L N_{G}(P)$ whose irreducible constituents all have degree divisible by $p$. Furthermore, the map $\chi \mapsto \chi^{*}$ is a bijection

$$
\operatorname{Irr}_{p^{\prime}}\left(B_{0}(G)\right) \rightarrow \operatorname{Irr}_{p^{\prime}}\left(B_{0}\left(L N_{G}(P)\right)\right) .
$$

Proof. (I) Let $(G, L)$ be a counterexample to the first part of the theorem with $|G| \cdot|G / L|$ as small as possible. 
(a) Here we show that $\boldsymbol{O}_{p^{\prime}}(G)=1$ and $\boldsymbol{N}_{G}(P)=P$. To this end, using Theorem 3.2 we can write $N_{G}(P)=P \times X$, where $X \leq N:=\boldsymbol{O}_{p^{\prime}}(G)$. Write $\bar{G}=G / N$ and use the bar convention. Hence $\bar{L}=L N / N, \bar{P}=P N / N$ and $N_{\bar{G}}(\bar{P})=\bar{P} \times \bar{X}=\bar{P}$, by elementary group theory. Now, $N \leq \operatorname{ker}(\chi)$. If $N>1$, then, considering $\chi$ as a character of $\bar{G}$, by induction we have that

$$
\chi_{\bar{L} N_{\bar{G}}(\bar{P})}=\chi_{\bar{L} \bar{P}}=\chi^{*}+\Delta,
$$

where $\chi^{*}$ is an irreducible character of $p^{\prime}$-degree in the principal block of $\bar{L} \bar{P}=$ $L P N / N$ and either $\Delta=0$ or $\Delta$ is a character of $L P N / N$ such that every irreducible constituent of $\Delta$ has degree divisible by $p$. Now, Lemma 4.2 applies, and we are done in this case. So we have that $N=1$ and that $N_{G}(P)=P$. Hence, every $p^{\prime}$-degree character of every subgroup $H$ with $P \leq H \leq G$ (or of every quotient $G / K$ of $G$ ) lies in the principal block of $H$ (of $G / K$ ) by the first main theorem of Brauer (Theorem (4.17) of [Navarro 1998]).

(b) Next we show that $L=1$. By Lemma 2.1, let $\theta \in \operatorname{Irr}(L)$ be $P$-invariant under $\chi$. Let $T=I_{G}(\theta)$ be the stabilizer of $\theta$ in $G$, and let $\psi \in \operatorname{Irr}(T \mid \theta)$ be the Clifford correspondent of $\chi$ over $\theta$. Assume that $T<G$. By the choice of $G$, we have that

$$
\psi_{L P}=\psi^{*}+\Xi
$$

where $\psi^{*}$ has $p^{\prime}$-degree and either $\Xi=0$ or the irreducible constituents of $\Xi$ have degree divisible by $p$. Now, we use Lemma 2.2 to conclude that we may assume that $\theta$ is $G$-invariant. By Corollary 2.4, we then have that $\theta$ has an extension $\tilde{\theta} \in \operatorname{Irr}(G)$. Now, by Gallagher's corollary [Isaacs 2006, Corollary (6.17)], we have that $\chi=\beta \tilde{\theta}$, for some $\beta \in \operatorname{Irr}(G / L)$. Now if $L \neq 1$, then the theorem holds for $G / L$, whence we have that $\beta_{P L}$ is the sum of a $p^{\prime}$-degree irreducible character $\beta^{*}$ of $P L / L$ (and hence linear) plus some character $\Delta$ of $P L / L$ such that all of its irreducible constituents have degree divisible by $p$, or $\Delta=0$. Then

$$
\chi_{L P}=\left(\beta^{*}\right) \tilde{\theta}_{L P}+\Delta \tilde{\theta}_{L P},
$$

and, using Gallagher's corollary, we see that we are done again. Hence $L=1$, as desired.

(c) Now we can show that $p=3, E:=\boldsymbol{F}^{*}(G)=\boldsymbol{E}(G)=S_{1} \times \cdots \times S_{n}$ with $S_{i} \cong \mathrm{PSL}_{2}\left(q_{i}\right)$ for some $q_{i}=3^{3^{a_{i}}}, a_{i}, n \geq 1$, and $E \triangleleft G \leq \operatorname{Aut}(E)$. Indeed, suppose that $K:=\boldsymbol{O}_{p}(G) \neq 1$. Since $|G / K|<|G|=|G / L|$, the first statement of the theorem holds for $(G, K)$ and so for $(G, L)$ as well (since $K P=P)$, contradicting the choice of $(G, L)$. Thus $\boldsymbol{O}_{p}(G)=1$. Since $\boldsymbol{O}_{p^{\prime}}(G)=1$, we have now that $\boldsymbol{F}(G)=1$ and so $E=\boldsymbol{F}^{*}(G)=\boldsymbol{E}(G)$. Next, $\boldsymbol{Z}(E) \leq \boldsymbol{F}(G)=1$, whence $E=S_{1} \times \cdots \times S_{n}$ is a direct product of nonabelian simple groups and $C_{G}(E)=1$, yielding $E \triangleleft G \leq \operatorname{Aut}(E)$. 
Since $N_{G}(P)=P$ and $p$ is odd, we have that $p=3$ and $S_{i} \cong \operatorname{PSL}_{2}\left(q_{i}\right)$ with $q_{i}=3^{3^{a_{i}}}$ by the main result of [Guralnick et al. 2004].

(d) Let $Q:=P \cap E \in \operatorname{Syl}_{p}(E)$ and write $Q=Q_{1} \times \cdots \times Q_{n}$ with $Q_{i} \in \operatorname{Syl}_{p}\left(S_{i}\right)$. Since $P$ is self-normalizing in $E P$, by [Navarro et al. 2007, Lemma 2.1(ii)], $\boldsymbol{C}_{\boldsymbol{N}_{E}(Q) / Q}(P)=1$. This in turn implies by Lemma 4.1 that $\boldsymbol{C}_{N_{S_{i}}\left(Q_{i}\right) / Q_{i}}\left(P_{i}\right)=1$ for $P_{i}:=N_{P}\left(S_{i}\right)$. It follows by Lemma 3.1(ii) that $P_{i}$ must induce the full subgroup $C_{3^{a_{i}}}$ of field automorphisms of $S_{i}$. Applying Lemma 3.1(iii) to $S_{i}$, we see that the $P_{i}$-invariant irreducible characters of $p^{\prime}$-degree of $S_{i}$ are $\alpha_{i}:=1_{S_{i}}$ and the two Weil characters $\eta_{i}^{ \pm}$of degree $\left(q_{i}-1\right) / 2$. Furthermore, for each $\alpha \in\left\{\alpha_{i}, \eta_{i}^{ \pm}\right\}, P_{i}$ fixes a unique irreducible constituent $\alpha^{*}$ of $\alpha_{Q_{i}}$, occurring with multiplicity one. Moreover, the map $\alpha \mapsto \alpha^{*}$ is a bijection between the set of irreducible $P_{i}$-invariant characters of $p^{\prime}$-degree of $S_{i}$ and that of $Q_{i}$.

(e) Since the theorem holds for $(G, E)$,

$$
\chi_{E P}=\chi^{*}+\Delta,
$$

where $\chi^{*} \in \operatorname{Irr}_{p^{\prime}}(E P)$ and all the irreducible constituents of $\Delta$ (if any) have degree divisible by $p$. In particular, $\theta:=\left(\chi^{*}\right)_{E}$ is irreducible. Write

$$
\theta=\theta_{1} \times \cdots \times \theta_{n},
$$

with $\theta_{i} \in \operatorname{Irr}_{p^{\prime}}\left(S_{i}\right)$. Since $\theta$ is $P$-invariant, it follows that $\theta_{i}$ is $P_{i}$-invariant of $p^{\prime}$-degree, and so $\theta_{i} \in\left\{\alpha_{i}, \eta_{i}^{ \pm}\right\}$by (d). As mentioned above,

$$
\left(\theta_{i}\right)_{Q_{i}}=\theta_{i}^{*}+\delta_{i},
$$

where $\theta_{i}^{*} \in \operatorname{Irr}\left(Q_{i}\right)$ is $P_{i}$-invariant and $\delta_{i}$ is a sum of non- $P_{i}$-invariant irreducible characters of $Q_{i}$. Setting

$$
\tilde{\theta}:=\theta_{1}^{*} \times \cdots \times \theta_{n}^{*},
$$

we see that each irreducible constituent of $\theta_{Q}-\tilde{\theta}$ is non- $P$-invariant and so must lie under an irreducible character of $P$ of degree divisible by $p$. But $p \nmid \theta(1)$. Hence $\theta_{P}$ contains a unique linear constituent which lies above $\tilde{\theta}$. Denote this constituent by $\theta^{*}$. We have shown that every irreducible constituent of $\theta_{P}-\theta^{*}=\left(\chi^{*}\right)_{P}-\theta^{*}$ is of degree divisible by $p$, whereas $\theta^{*}(1)=1$.

(f) It remains to show that every irreducible constituent of $\Delta_{P}$ has degree divisible by $p$. Assume the contrary: $\Delta_{P}$ contains a linear constituent $\lambda$, and write

$$
\lambda_{Q}=\lambda_{1} \times \cdots \times \lambda_{n},
$$

with $\lambda_{i} \in \operatorname{Irr}\left(Q_{i}\right)$. Let $\gamma \in \operatorname{Irr}(E P)$ be an irreducible constituent of $\Delta$ that contains $\lambda$ upon restriction to $P$. Also, let

$$
\beta=\beta_{1} \times \cdots \times \beta_{n} \in \operatorname{Irr}(E)
$$


lie under $\gamma$ and above $\lambda_{Q}$. Since $E \triangleleft G$ and both $\theta$ and $\beta$ are irreducible constituents of $\chi_{E}, \beta$ is $G$-conjugate to $\theta$. Recall that $\theta=\theta_{1} \times \cdots \times \theta_{n}$, with $\theta_{i} \in\left\{\alpha_{i}, \eta_{i}^{ \pm}\right\}$. Also, note that the set $\left\{\alpha_{i}, \eta_{i}^{ \pm}\right\}$is $\operatorname{Aut}\left(S_{i}\right)$-invariant. It follows that $\beta_{i} \in\left\{\alpha_{i}, \eta_{i}^{ \pm}\right\}$. As mentioned in (d), $\left(\beta_{i}\right)_{Q_{i}}$ contains a unique $P_{i}$-invariant irreducible constituent $\beta_{i}^{*}$, and each irreducible constituent of $\left(\beta_{i}\right)_{Q_{i}}-\beta_{i}^{*}$ is non- $P_{i}$-invariant. Denoting

$$
\tilde{\beta}:=\beta_{1}^{*} \times \cdots \times \beta_{n}^{*},
$$

we see that no irreducible constituent of $\beta_{Q}-\tilde{\beta}$ can be invariant under $P$. But $\lambda_{Q}$ lies under $\beta_{Q}$ and is $P$-invariant. Hence $\lambda_{Q}=\tilde{\beta}$ and $\lambda_{i}=\beta_{i}^{*}$.

(g) Now we consider two cases.

Case 1: $\beta$ is not $P$-invariant. In this case, there is some $g \in P$ such that $\beta^{g} \neq \beta$. Then $\beta^{g}$ lies above $\left(\lambda_{Q}\right)^{g}=\lambda_{Q}$ and under $\gamma$. Writing $\beta^{g}=\beta_{1}^{\prime} \times \cdots \times \beta_{n}^{\prime}$ and arguing as in (f), we see that $\beta_{i}^{\prime} \in\left\{\alpha_{i}, \eta_{i}^{ \pm}\right\}$and, moreover,

$$
\beta_{i}^{*}=\lambda_{i}=\left(\beta_{i}^{\prime}\right)^{*} .
$$

As mentioned in (d), the map $\alpha \mapsto \alpha^{*}$ is a bijection. It follows that $\beta_{i}=\beta_{i}^{\prime}$ and so $\beta=\beta^{g}$, a contradiction.

Case 2: $\beta$ is $P$-invariant. Then, by Corollary $2.4, \beta$ extends to $\hat{\beta} \in \operatorname{Irr}(E P)$. Since $\gamma$ lies above $\beta$, by Gallagher's corollary we have that $\gamma=\hat{\beta} \mu$, where $\mu \in \operatorname{Irr}(P / Q)$ is considered as a character of $E P / E$. Note that $p \mid \gamma(1)$, as $\gamma$ is an irreducible constituent of $\Delta$. On the other hand, $p \nmid \hat{\beta}(1)=\beta(1)$. It follows that $p \mid \mu(1)$. As shown in (f), no irreducible constituent of

$$
\hat{\beta}_{Q}-\lambda_{Q}=\beta_{Q}-\lambda_{Q}
$$

can be $P$-invariant. Hence $\lambda$ is the unique linear constituent of $\hat{\beta}_{P}$. Certainly, $\mu \lambda$ is irreducible over $P$ and nonlinear. Furthermore, again as shown in (f), every irreducible constituent of

$$
\left(\gamma_{P}-\mu \lambda\right)_{Q}=\mu(1) \cdot\left(\beta_{Q}-\lambda_{Q}\right)
$$

is non- $P$-invariant and so must lie under an irreducible $P$-character of degree divisible by $p$. Thus the degree of every irreducible constituent of $\gamma_{P}-\mu \lambda$ is divisible by $p$, and the same is true for $\mu \lambda \in \operatorname{Irr}(P)$. Consequently, the linear character $\lambda$ cannot be a constituent of $\gamma_{P}$, again a contradiction.

Thus we have completed the proof of the first statement of the theorem.

(II) Now we prove that our map $\chi \mapsto \chi^{*}$ is a bijection. Recall that $\boldsymbol{O}_{p^{\prime}}\left(\boldsymbol{N}_{G}(P)\right) \leq$ $\boldsymbol{O}_{p^{\prime}}(G)$ by Theorem 3.2 and that $\boldsymbol{O}_{p^{\prime}}(G)$ is contained in the kernel of any $\psi \in$ $\operatorname{Irr}\left(B_{0}(G)\right)$. Modding out by $\boldsymbol{O}_{p^{\prime}}(G)$, we may assume that $\boldsymbol{O}_{p^{\prime}}(G)=1$ and so $N_{G}(P)=P$. Hence the principal block is the only block of maximal defect of $G$, and the same is true for $L P$. Since all the nonabelian composition factors of $G$ 
of order divisible by $p$ are $\mathrm{PSL}_{2}\left(3^{3^{a}}\right)$ with $a \geq 1$, we know by [Isaacs et al. 2007, Theorem A] that the McKay conjecture is true for $G$ and for $L P$. Hence

$$
\left|\operatorname{Irr}_{p^{\prime}}(G)\right|=\left|\operatorname{Irr}_{p^{\prime}}\left(N_{G}(P)\right)\right|=\left|\operatorname{Irr}\left(P / P^{\prime}\right)\right|=\left|\operatorname{Irr}_{p^{\prime}}\left(N_{L P}(P)\right)\right|=\left|\operatorname{Irr}_{p^{\prime}}(L P)\right| .
$$

Now, if $\delta \in \operatorname{Irr}_{p^{\prime}}(L P)$, then some irreducible constituent $\chi$ of $\delta^{G}$ has $p^{\prime}$-degree. Therefore $\chi_{L P}$ contains $\delta$ and, by the first statement of the theorem, we necessarily have that $\chi^{*}=\delta$. Thus the map $\chi \mapsto \chi^{*}$ is surjective, and therefore injective.

The proof of Corollary B, which we restate below, is now immediate.

Corollary B. Let $G$ be a finite group, let $p$ be odd, and let $P \in \operatorname{Syl}_{p}(G)$. Suppose that $N_{G}(P)=P$. Then there is a natural bijection $\chi \mapsto \chi^{*}$ between $\operatorname{Irr}_{p^{\prime}}(G)$ and the linear characters of $P$. In fact, if $\chi \in \operatorname{Irr}_{p^{\prime}}(G)$ and $\lambda \in \operatorname{Irr}(P)$ is linear, then $\chi$ and $\lambda$ correspond under the bijection if and only if

$$
\chi_{P}=\lambda+\Delta,
$$

where $\Delta$ is either zero or a character whose irreducible constituents all have degree divisible by $p$. This happens if and only if

$$
\lambda^{G}=\chi+\Xi
$$

where $\Xi$ is either zero or a character whose irreducible constituents all have degree divisible by $p$.

Proof. If $N_{G}(P)=P$, then the principal block is the unique block of maximal defect by Brauer's first main theorem. Hence, the first part of the corollary follows from Theorem 4.3 by letting $L=1$. For the second part, if $\lambda \in \operatorname{Irr}(P)$ is linear, then $\lambda^{G}$ has degree not divisible by $p$, and therefore $\lambda^{G}$ has a constituent $\chi \in \operatorname{Irr}_{p^{\prime}}(G)$. Then $\left[\chi_{P}, \lambda\right] \neq 0$ and it follows that necessarily $\lambda=\chi^{*}$. It also follows that $\chi$ is unique, because our map is injective.

Next is Corollary C.

Corollary C. Let $G$ be a finite group, let $p$ be odd, and let $P \in \operatorname{Syl}_{p}(G)$.

(a) $N_{G}(P)=P$ if and only if

$$
\left(1_{P}\right)^{G}=1_{G}+\Xi
$$

where $\Xi$ is either zero or a character whose irreducible constituents all have degree divisible by $p$.

(b) $N_{G}(P)=P C_{G}(P)$ if and only $1_{G}$ is the only irreducible constituent of $\left(1_{P C_{G}(P)}\right)^{G}$ that belongs to $\operatorname{Irr}_{p^{\prime}}\left(B_{0}(G)\right)$. 
Proof. Both proofs are very similar. We start with (a). One implication follows from Corollary B. Assume now that

$$
\left(1_{P}\right)^{G}=1_{G}+\Xi,
$$

where $\Xi$ is either zero or a character whose irreducible constituents all have degree divisible by $p$, but $N:=N_{G}(P)>P$. Then there exists a nonprincipal character $\gamma \in \operatorname{Irr}(N / P)$, which can be viewed as an $N$-character. Since $\gamma$ has $p^{\prime}$-degree (because $N / P$ is a $p^{\prime}$-group), it follows that $\gamma^{G}$ possesses an irreducible constituent $\chi \in \operatorname{Irr}_{p^{\prime}}(G)$. Now, $\chi$ lies over $\gamma \neq 1_{N}$ and therefore $1_{G} \neq \chi$ lies over $1_{P}$, a contradiction.

Next, we prove (b). Write $C=\boldsymbol{C}_{G}(P)$. One implication follows from Theorem 4.3. Assume now that $1_{G}$ is the unique irreducible constituent of $\left(1_{P C}\right)^{G}$ that belongs to $\operatorname{Irr}_{p^{\prime}}\left(B_{0}(G)\right)$ and that $N:=N_{G}(P)>P C$. Then there exists a nonprincipal character $\gamma \in \operatorname{Irr}(N / P C)$, which can be viewed as an $N$-character. Since $N$ is $p$-solvable, and $\boldsymbol{O}_{p^{\prime}}(N) \leq C \leq \operatorname{ker} \gamma$, it follows that $\gamma$ lies in the principal block of $N$ by [Navarro 1998, Theorem (10.20)]. Also, $\gamma$ has $p^{\prime}$-degree, because $N / P C$ is a $p^{\prime}$-group. If $b$ is now the principal block of $N$, we know that $b^{G}=B=B_{0}(G)$ is the principal block of $G$, by Brauer's third main theorem [loc. cit., Theorem (6.7)]. Write

$$
\left(\gamma^{G}\right)_{B}=\sum_{\chi \in \operatorname{Irr}(B)}\left[\gamma^{G}, \chi\right] \chi .
$$

(This is called the $B$-part of $\gamma^{G}$; see page 72 of [loc. cit.].) Now, by [loc. cit., Corollary (6.4)], we have that

$$
1=\left(\gamma^{G}(1)\right)_{p}=\left(\left(\gamma^{G}\right)_{B}(1)\right)_{p},
$$

where $n_{p}$ is the largest power of $p$ dividing the integer $n$. It then follows that some irreducible constituent $\chi$ of $\gamma^{G}$ lies in $\operatorname{Irr}_{p^{\prime}}(B)$. We now have that $\chi$ lies over $\gamma$ and therefore over $1_{P C}$. Since $\gamma \neq 1_{N}$, it follows that $\chi \neq 1_{G}$, and this is a contradiction.

\section{5. $p$-solvable groups}

Our proof of Theorem D is short but uses deep character theory of $p$-solvable groups. We assume that the reader is familiar with $\pi$-special characters (i.e., the characters of $\pi$-degree whose subnormal irreducible constituents have determinantal $\pi$-order; see [Gajendragadkar 1979]).

Lemma 5.1. Suppose that $L \triangleleft G, P \in \operatorname{Syl}_{p}(G)$ and $N_{G / L}(P L / L)=P L / L$. Assume that $G / L$ is $p$-solvable. Let $\theta \in \operatorname{Irr}(L)$ be $P$-invariant and $p^{\prime}$-special. Then there exists a unique $\hat{\theta} \in \operatorname{Irr}(G \mid \theta)$ such that $\hat{\theta}$ is $p^{\prime}$-special. 
Proof. We argue by induction on $|G: L|$. Let $K / L$ be a chief factor of $G$, and notice that $G / K$ has a self-normalizing Sylow $p$-subgroup, by elementary group theory. Assume first that $K / L$ is a $p$-group, and let $\eta \in \operatorname{Irr}(K \mid \theta)$ be the unique $p^{\prime}$-special character lying over $\theta$, by using Proposition 4.3 of [Gajendragadkar 1979]. By uniqueness, $\eta$ is $P$-invariant, and by induction, there is a unique $p^{\prime}$-special character $\hat{\eta} \in \operatorname{Irr}(G)$ that lies over $\eta$ (and therefore over $\theta$ ). Now, if $\hat{\theta}$ is any other $p^{\prime}$-special character of $G$ lying over $\theta$ and $\psi \in \operatorname{Irr}(K)$ lies under $\hat{\theta}$ and over $\theta$, we have that $\psi$ is $p^{\prime}$-special by Proposition 4.1 of [Gajendragadkar 1979], and therefore $\psi=\eta$, by uniqueness. But in this case, $\hat{\theta}=\hat{\eta}$, by using the inductive hypothesis.

Suppose finally that $K / L$ is a $p^{\prime}$-group. Then $C_{K / L}(P L / L)=1$, using that $P L / L$ is self-normalizing. Hence, by Problem (13.10) of [Isaacs 2006], there exists a unique $P$-invariant $\tau \in \operatorname{Irr}(K \mid \theta)$. Also, $\tau$ is $p^{\prime}$-special by Lemma 4.4 of [Gajendragadkar 1979]. By induction, there exists a unique $p^{\prime}$-special character $\hat{\tau}$ lying over $\tau$ (and therefore over $\theta$ ). Suppose now that $\gamma \in \operatorname{Irr}(G)$ is any other $p^{\prime}$-special character lying over $\theta$. By Lemma 2.1, let $\phi \in \operatorname{Irr}(K)$ be $P$-invariant under $\gamma$, and, by Theorem (13.27) of [Isaacs 2006], let $\rho \in \operatorname{Irr}(L)$ be $P$-invariant under $\phi$. Then $\rho$ and $\theta$ are $P$-invariant and lie under $\gamma$, so $\rho=\theta$ by Lemma 2.1. Then $\phi=\tau$ by the uniqueness of $\tau$, and hence $\gamma=\hat{\tau}$ by induction.

We restate Theorem $\mathrm{D}$ for the reader's convenience.

Theorem D. Let $G$ be a finite $p$-solvable group, and let $P \in \operatorname{Syl}_{p}(G)$. Suppose that $\boldsymbol{N}_{G}(P)=P \boldsymbol{C}_{G}(P)$, and let $N=\boldsymbol{O}_{p^{\prime}}(G)$. Let $\operatorname{Irr}_{P}(N)$ be the set of $P$-invariant $\theta \in \operatorname{Irr}(N)$. Then for every $\theta \in \operatorname{Irr}_{P}(N)$ and linear $\lambda \in \operatorname{Irr}\left(P / P^{\prime}\right)$, there is a canonically defined character

$$
\lambda \star \theta \in \operatorname{Irr}_{p^{\prime}}(G)
$$

Furthermore, the map

$$
\operatorname{Irr}\left(P / P^{\prime}\right) \times \operatorname{Irr}_{P}(N) \rightarrow \operatorname{Irr}_{p^{\prime}}(G)
$$

given by $(\lambda, \theta) \mapsto \lambda \star \theta$ is a bijection. As a consequence, $\boldsymbol{N}_{G}(P)=P \times \boldsymbol{C}_{N}(P)$, and if $\theta^{*} \in \operatorname{Irr}\left(\boldsymbol{C}_{N}(P)\right)$ is the Glauberman correspondent of $\theta \in \operatorname{Irr}_{P}(N)$, then the map

$$
\lambda \times \theta^{*} \mapsto \lambda \star \theta
$$

is a natural bijection $\operatorname{Irr}_{p^{\prime}}\left(N_{G}(P)\right) \rightarrow \operatorname{Irr}_{p^{\prime}}(G)$. Also, if $\theta=1_{N}$ and $\lambda \in \operatorname{Irr}\left(P / P^{\prime}\right)$, then $\lambda \times \theta^{*}$ is the unique linear constituent of $(\lambda \star \theta)_{N_{G}(P)}$.

Proof. By Theorem 3.2, we can write $N_{G}(P)=P \times X$, where $X:=C_{N}(P)$. Let $\lambda \in \operatorname{Irr}(P)$ be linear and let $\theta \in \operatorname{Irr}_{P}(N)$. Since $P \cap N=1$, we trivially have that $\lambda$ extends to $P N$. Now, by Theorem 2.1 of [Isaacs and Navarro 2008] (or see Corollary 2.2 of [Isaacs and Navarro 2001] for a self-contained proof), there exists a maximal subgroup $P \subseteq W \subseteq G$ such that $\lambda$ extends to $W$. Hence $P N \subseteq W$. Now, 
by elementary character theory, let $\hat{\lambda} \in \operatorname{Irr}(W)$ be the unique linear character of $p$-power order that extends $\lambda$. Now, $N_{W / N}(P N / N)=P N / N$, and by Lemma 5.1, there exists a unique $p^{\prime}$-special $\hat{\theta} \in \operatorname{Irr}(W)$ lying over $\theta$. Now, by Theorem 2.2 of [Isaacs and Navarro 2008] and Theorem C of [Navarro 1997] we have that

$$
\lambda \star \theta:=(\hat{\theta} \hat{\lambda})^{G} \in \operatorname{Irr}(G) .
$$

Notice that $\lambda \star \theta$ has $p^{\prime}$-degree, because $\hat{\theta}$ has $p^{\prime}$-degree and $|G: W|$ is not divisible by $p$. (We notice for the record that $(\lambda \star \theta)_{W}$ contains $\hat{\theta} \hat{\lambda}$, and therefore, when restricted to $N$, we have that $(\lambda \star \theta)$ lies over $\theta$. It is not in general true that $\lambda \star \theta$ lies over $\lambda$, on the other hand.)

We have now defined a map

$$
\operatorname{Irr}\left(P / P^{\prime}\right) \times \operatorname{Irr}_{P}(N) \rightarrow \operatorname{Irr}_{p^{\prime}}(G)
$$

given by $(\lambda, \theta) \mapsto \lambda \star \theta$.

Next we show that our map is surjective. Let $\chi \in \operatorname{Irr}_{p^{\prime}}(G)$. By Theorem 3.6 of [Isaacs and Navarro 2008], we have that $\chi$ is a satellite of some $\psi \in B_{p}(G)$ of $p^{\prime}$-degree (see Section 3 of [Isaacs and Navarro 2008] for the necessary definitions). In other words, this means that there is some linear character $\delta \in \operatorname{Irr}(P)$ and a $p^{\prime}$-special character $\alpha \in \operatorname{Irr}(U)$, where $U$ is the maximal subgroup of $G$ to which $\delta$ extends, such that

$$
\chi=(\hat{\delta} \alpha)^{G},
$$

where the order of $\hat{\delta}$ is a $p$-power and $\hat{\delta}$ extends $\delta$. Now, $\alpha_{N}$ contains a (unique) $P$-invariant character $\mu \in \operatorname{Irr}_{P}(N)$ by Lemma 2.1, and it follows that $\alpha$ is the unique $p^{\prime}$-special character of $U$ lying over $\mu$ by Lemma 5.1. It follows then that $\chi=\delta \star \mu$, and, therefore, that our map is surjective.

Recall that the Glauberman correspondence [Isaacs 2006, Theorem (13.1)] provides a natural bijection

$$
\operatorname{Irr}_{P}(N) \rightarrow \operatorname{Irr}\left(C_{N}(P)\right) .
$$

Since the McKay conjecture is true for $p$-solvable groups (see for instance [Isaacs et al. 2007]) we have that

$$
\left|\operatorname{Irr}_{p^{\prime}}(G)\right|=\left|\operatorname{Irr}_{p^{\prime}}\left(N_{G}(P)\right)\right|=\left|\operatorname{Irr}\left(P / P^{\prime}\right)\right|\left|\operatorname{Irr}\left(C_{N}(P)\right)\right|=\left|\operatorname{Irr}\left(P / P^{\prime}\right)\right|\left|\operatorname{Irr}_{P}(N)\right| .
$$

It then follows that our map is bijective.

In the case where $\theta=1_{N}$, the second part of the theorem easily follows from Theorem 3.1 of [Isaacs and Navarro 2008] applied in the group $G / N$.

Under the hypothesis of the previous theorem, we notice that the blocks with defect group $P \in \operatorname{Syl}_{p}(G)$ of $G$ can be parametrized by the $P$-invariant irreducible characters of $N=\boldsymbol{O}_{p^{\prime}}(G)$. The fact that in this case $\operatorname{Irr}_{p^{\prime}}(G \mid \theta)$ and $\operatorname{Irr}_{p^{\prime}}\left(\boldsymbol{N}_{G}(P) \mid \theta^{*}\right)$ 
have the same cardinality is a consequence of [Okuyama and Wajima 1980]. Our hypothesis, however, allows us to obtain a canonical bijection in our case.

\section{A non- $p$-solvable example}

To finish the paper, it might be interesting to show the reader how to construct a natural bijection $\operatorname{Irr}_{p^{\prime}}(G) \rightarrow \operatorname{Irr}_{p^{\prime}}\left(N_{G}(P)\right)$ in the paradigmatic case where $G=$ $\mathrm{SL}_{2}\left(3^{3^{a}}\right) \cdot C_{3^{a}}$ and $p=3$, with $P \in \operatorname{Syl}_{p}(G)$ as usual.

Let $S:=[G, G]=\operatorname{SL}_{2}(q)$ with $q=3^{3^{a}}$, and let $\sigma$ denote the field automorphism of $S$ of order $t:=3^{a}$, so that $G=S \rtimes\langle\sigma\rangle$. Using [Digne and Michel 1991, Table 2], it is easy to check that $\operatorname{Irr}_{p^{\prime}}(S)$ contains exactly six $\sigma$-invariant characters: $1_{S}$, two Weil characters $\eta_{1,2}$ of degree $(q-1) / 2$ (denoted $\chi_{b}^{ \pm}$in [Isaacs et al. 2007, §15]), two Weil characters $\xi_{1,2}$ of degree $(q+1) / 2$ (denoted $\chi_{a}^{ \pm}$in that work), and a unique rational-valued character $\psi$ of degree $q-1$. Here, the three former characters are nonfaithful, and the three latter ones are faithful. Furthermore, one can label $\xi_{1,2}$ such that

$$
\xi_{i}(x)=\eta_{i}(x)+1
$$

for any element $x \in S$ of order 3 and $i=1,2$. Since $G / S$ is cyclic (and generated by $\sigma$ ), it follows that all these 6 characters extend to $G$, and the $6 t$ extensions are precisely the characters in $\operatorname{Irr}_{p^{\prime}}(G)$. In particular, $1_{S}$ extends to $\lambda_{j}, 1 \leq j \leq t$, with $\lambda_{1}=1_{G}$. Next, we will single out a "canonical" extension for each of the remaining five characters of $S$. As shown in [Navarro and Tiep 2014, §3], $G$ embeds in $H:=\mathrm{Sp}_{2 t}(3)$ in such a way that $\eta_{i}$ extends to a Weil character of $H$ that takes value 1 at $\sigma$. We will denote the restriction of this character of $H$ to $G$ by $\tilde{\eta}_{i}$, so that

$$
\tilde{\eta}_{i}(\sigma)=1, \quad i=1,2 .
$$

Likewise, $\xi_{i}$ extends to a Weil character of $H$ that takes value 2 at $\sigma$, and we will denote the restriction of this character of $H$ to $G$ by $\tilde{\xi}_{i}$, so that

$$
\tilde{\xi}_{i}(\sigma)=2, \quad i=1,2 .
$$

Finally, by [Navarro and Tiep 2008, Corollary 2.2], there is a unique rational-valued extension $\tilde{\psi}$ of $\psi$ to $G$.

Let $1_{Z}$ and $v$ denote the two linear characters of $Z:=Z(G) \cong C_{2}$. For any $\gamma \in \operatorname{Irr}(Z)$, let $\operatorname{Irr}_{p^{\prime}}(G \mid \gamma)$ denote the set of characters $\chi \in \operatorname{Irr}_{p^{\prime}}(G)$ that lie above $\gamma$, and similarly for $N:=N_{G}(P)=P \times Z$. Now we see that

$$
\begin{aligned}
\operatorname{Irr}_{p^{\prime}}\left(G \mid 1_{Z}\right) & =\left\{\lambda_{j}, \tilde{\eta}_{i} \lambda_{j} \mid 1 \leq i \leq 2,1 \leq j \leq t\right\}, \\
\operatorname{Irr}_{p^{\prime}}(G \mid v) & =\left\{\tilde{\psi} \lambda_{j}, \tilde{\xi}_{i} \lambda_{j} \mid 1 \leq i \leq 2,1 \leq j \leq t\right\} .
\end{aligned}
$$


Moreover, the first set is contained in the principal 3-block $B_{0}(G)$ of $G$ and the second set is contained in the other 3-block of maximal defect $B_{1}(G)$ of $G$. Theorem A yields a natural correspondence $\operatorname{Irr}_{p^{\prime}}\left(B_{0}(G)\right) \rightarrow \operatorname{Irr}_{p^{\prime}}\left(B_{0}(N)\right)$. To get a natural correspondence $\operatorname{Irr}_{p^{\prime}}\left(B_{1}(G)\right) \rightarrow \operatorname{Irr}_{p^{\prime}}\left(B_{1}(N)\right)$, it therefore suffices to define a natural correspondence between $\operatorname{Irr}_{p^{\prime}}\left(G \mid 1_{Z}\right)=\operatorname{Irr}_{p^{\prime}}\left(B_{0}(G)\right)$ and $\operatorname{Irr}_{p^{\prime}}(G \mid v)=\operatorname{Irr}_{p^{\prime}}\left(B_{1}(G)\right)$, which can be given by

$$
\lambda_{j} \mapsto \tilde{\psi} \lambda_{j}, \quad \tilde{\eta}_{i} \lambda_{j} \mapsto \tilde{\xi}_{i} \lambda_{j},
$$

and a natural correspondence between $\operatorname{Irr}_{p^{\prime}}\left(N \mid 1_{Z}\right)=\operatorname{Irr}_{p^{\prime}}\left(B_{0}(N)\right)$ and $\operatorname{Irr}_{p^{\prime}}(N \mid v)=$ $\operatorname{Irr}_{p^{\prime}}\left(B_{1}(N)\right)$, which can be given by

$$
\mu \times 1_{Z} \mapsto \mu \times v
$$

for all $\mu \in \operatorname{Irr}\left(P / P^{\prime}\right)$.

Note that an equivariant bijection $\pi: \operatorname{Irr}_{p^{\prime}}(S) \rightarrow \operatorname{Irr}_{p^{\prime}}\left(N_{S}(P \cap S)\right)$ was constructed in [Isaacs et al. 2007, (15F)]. Choosing $\pi\left(\chi_{a}^{ \pm}\right)$and $\pi\left(\chi_{b}^{ \pm}\right)$suitably, one can check that $\pi$ extends (from $S$ to $G$ ) to our bijection $\operatorname{Irr}_{p^{\prime}}(G) \rightarrow \operatorname{Irr}_{p^{\prime}}\left(N_{G}(P)\right.$ ).

\section{Acknowledgements}

The authors are grateful to the referee for careful reading and helpful comments that greatly improved the exposition of the paper.

\section{References}

[Digne and Michel 1991] F. Digne and J. Michel, Representations of finite groups of Lie type, London Mathematical Society Student Texts 21, Cambridge University Press, 1991. MR 92g:20063 Zbl 0815.20014

[Gajendragadkar 1979] D. Gajendragadkar, "A characteristic class of characters of finite $\pi$-separable groups”, J. Algebra 59:2 (1979), 237-259. MR 82b:20012 Zbl 0426.20007

[Guralnick et al. 2004] R. M. Guralnick, G. Malle, and G. Navarro, "Self-normalizing Sylow subgroups", Proc. Amer. Math. Soc. 132:4 (2004), 973-979. MR 2004m:20043 Zbl 1049.20010

[Huppert 1967] B. Huppert, Endliche Gruppen, I, Die Grundlehren der Mathematischen Wissenschaften 134, Springer, Berlin-New York, 1967. MR 37 \#302 Zbl 0217.07201

[Isaacs 2006] I. M. Isaacs, Character theory of finite groups, AMS Chelsea, Providence, RI, 2006. MR 2270898 Zbl 1119.20005

[Isaacs 2008] I. M. Isaacs, Finite group theory, Graduate Studies in Mathematics 92, Amer. Math. Soc., Providence, RI, 2008. MR 2009e:20029 Zbl 1169.20001

[Isaacs and Navarro 2001] I. M. Isaacs and G. Navarro, "Characters of $p^{\prime}$-degree of $p$-solvable groups”, J. Algebra 246:1 (2001), 394-413. MR 2002m:20011 Zbl 0998.20008

[Isaacs and Navarro 2008] I. M. Isaacs and G. Navarro, "Character sums and double cosets", $J$. Algebra 320:10 (2008), 3749-3764. MR 2009i:20019 Zbl 1189.20012

[Isaacs et al. 2007] I. M. Isaacs, G. Malle, and G. Navarro, "A reduction theorem for the McKay conjecture”, Invent. Math. 170:1 (2007), 33-101. MR 2008h:20016 Zbl 1138.20010 
[Kondratiev and Mazurov 2003] A. S. Kondratiev and V. D. Mazurov, "2-signalizers of finite simple groups", Algebra Logika 42:5 (2003), 594-623. In Russian; translated in Algebra Logic 42:5 (2003), 333-348. MR 2004j:20024 Zbl 1067.20015

[Malle and Navarro 2012] G. Malle and G. Navarro, "Characterizing normal Sylow $p$-subgroups by character degrees”, J. Algebra 370 (2012), 402-406. MR 2966846 Zbl 1277.20010

[Navarro 1997] G. Navarro, "New properties of the $\pi$-special characters", J. Algebra 187:1 (1997), 203-213. MR 97m:20008 Zbl 0890.20010

[Navarro 1998] G. Navarro, Characters and blocks of finite groups, London Mathematical Society Lecture Note Series 250, Cambridge University Press, 1998. MR 2000a:20018 Zbl 0903.20004

[Navarro 2003] G. Navarro, "Linear characters of Sylow subgroups", J. Algebra 269:2 (2003), 589-598. MR 2004m:20019 Zbl 1037.20007

[Navarro 2004] G. Navarro, "The McKay conjecture and Galois automorphisms", Ann. of Math. (2) 160:3 (2004), 1129-1140. MR 2005m:20022 Zbl 1079.20010

[Navarro and Späth 2014] G. Navarro and B. Späth, "On Brauer's height zero conjecture”, J. Eur. Math. Soc. (JEMS) 16:4 (2014), 695-747. MR 3191974 Zbl 06293942

[Navarro and Tiep 2008] G. Navarro and P. H. Tiep, "Rational irreducible characters and rational conjugacy classes in finite groups”, Trans. Amer. Math. Soc. 360:5 (2008), 2443-2465. MR 2008k:20014 Zbl 1137.20009

[Navarro and Tiep 2014] G. Navarro and P. H. Tiep, "Brauer characters and rationality", Math. Z. 276:3-4 (2014), 1101-1112. MR 3175172 Zbl 06304104

[Navarro et al. 2007] G. Navarro, P. H. Tiep, and A. Turull, " $p$-rational characters and self-normalizing Sylow p-subgroups”, Represent. Theory 11 (2007), 84-94. MR 2008a:20018 Zbl 1146.20005

[Okuyama and Wajima 1980] T. Okuyama and M. Wajima, "Character correspondence and $p$-blocks of p-solvable groups”, Osaka J. Math. 17:3 (1980), 801-806. MR 82a:20013 Zbl 0446.20003

[Späth 2013] B. Späth, "A reduction theorem for the Alperin-McKay conjecture", J. Reine Angew. Math. 680 (2013), 153-189. MR 3100954 Zbl 1283.20006

Communicated by David Benson

Received 2014-01-31 Revised 2014-06-17 Accepted 2014-08-25

gabriel.navarro@uv.es Departamento de Álgebra, Universitat de València, Doctor Moliner, 50, 46100 Burjassot, València, Spain

tiep@math.arizona.edu Department of Mathematics, University of Arizona, 617 North Santa Rita Avenue, Tucson, AZ 85721, United States

carolina.vallejo@uv.es

Departamento de Álgebra, Universitat de València, Doctor Moliner, 50, 46100 Burjassot, València, Spain 


\section{Algebra \& Number Theory}

msp.org/ant

\section{EDITORS}

MANAGING EDITOR

Bjorn Poonen

Massachusetts Institute of Technology

Cambridge, USA

\author{
EDITORIAL BOARD CHAIR \\ David Eisenbud \\ University of California \\ Berkeley, USA
}

BOARD OF EDITORS

Georgia Benkart

Dave Benson

Richard E. Borcherds

John H. Coates

J-L. Colliot-Thélène

Brian D. Conrad

Hélène Esnault

Hubert Flenner

Edward Frenkel

Andrew Granville

Joseph Gubeladze

Roger Heath-Brown

Craig Huneke

János Kollár

Yuri Manin

Barry Mazur

Philippe Michel

Susan Montgomery
University of Wisconsin, Madison, USA

University of Aberdeen, Scotland

University of California, Berkeley, USA

University of Cambridge, UK

CNRS, Université Paris-Sud, France

University of Michigan, USA

Freie Universität Berlin, Germany

Ruhr-Universität, Germany

University of California, Berkeley, USA

Université de Montréal, Canada

San Francisco State University, USA

Oxford University, UK

University of Virginia, USA

Princeton University, USA

Northwestern University, USA

Harvard University, USA

École Polytechnique Fédérale de Lausanne

University of Southern California, USA
Shigefumi Mori

Raman Parimala

Jonathan Pila

Anand Pillay

Victor Reiner

Peter Sarnak

Joseph H. Silverman

Michael Singer

Vasudevan Srinivas

J. Toby Stafford

Bernd Sturmfels

Richard Taylor

Ravi Vakil

Michel van den Bergh

Marie-France Vignéras

Kei-Ichi Watanabe

Efim Zelmanov

Shou-Wu Zhang
RIMS, Kyoto University, Japan

Emory University, USA

University of Oxford, UK

University of Notre Dame, USA

University of Minnesota, USA

Princeton University, USA

Brown University, USA

North Carolina State University, USA

Tata Inst. of Fund. Research, India

University of Michigan, USA

University of California, Berkeley, USA

Harvard University, USA

Stanford University, USA

Hasselt University, Belgium

Université Paris VII, France

Nihon University, Japan

University of California, San Diego, USA

Princeton University, USA

\section{PRODUCTION}

production@msp.org

Silvio Levy, Scientific Editor

See inside back cover or msp.org/ant for submission instructions.

The subscription price for 2014 is US \$225/year for the electronic version, and $\$ 400 /$ year ( $\$ 55$, if shipping outside the US) for print and electronic. Subscriptions, requests for back issues and changes of subscribers address should be sent to MSP.

Algebra \& Number Theory (ISSN 1944-7833 electronic, 1937-0652 printed) at Mathematical Sciences Publishers, 798 Evans Hall \#3840, c/o University of California, Berkeley, CA 94720-3840 is published continuously online. Periodical rate postage paid at Berkeley, CA 94704, and additional mailing offices.

ANT peer review and production are managed by EditFLOW ${ }^{\circledR}$ from MSP.

\section{PUBLISHED BY}

mathematical sciences publishers

nonprofit scientific publishing

http://msp.org/

(C) 2014 Mathematical Sciences Publishers 


\section{Algebra \& Number Theory}

Volume $8 \quad$ No. $8 \quad 2014$

Relative cohomology of cuspidal forms on PEL-type Shimura varieties

KAI-WEN LAN and BENOÎT STROH

$\ell$-modular representations of unramified $p$-adic $\mathrm{U}(2,1)$

1801

ROBERT JAMES KURINCZUK

McKay natural correspondences on characters

Gabriel Navarro, Pham HuU Tiep and Carolina Vallejo

KAREL CASTEELS

Twisted Bhargava cubes

WEE TECK GAN and Gordan SAVIN

Proper triangular $\mathbb{G}_{a}$-actions on $\mathbb{A}^{4}$ are translations

AdRIEN Dubouloz, DAVID R. FInSTON and ImAD JARADAT

1985

Multivariate Apéry numbers and supercongruences of rational functions ARMIN STRAUB

The image of Carmichael's $\lambda$-function

Kevin Ford, Florian LuCA and Carl Pomerance 